
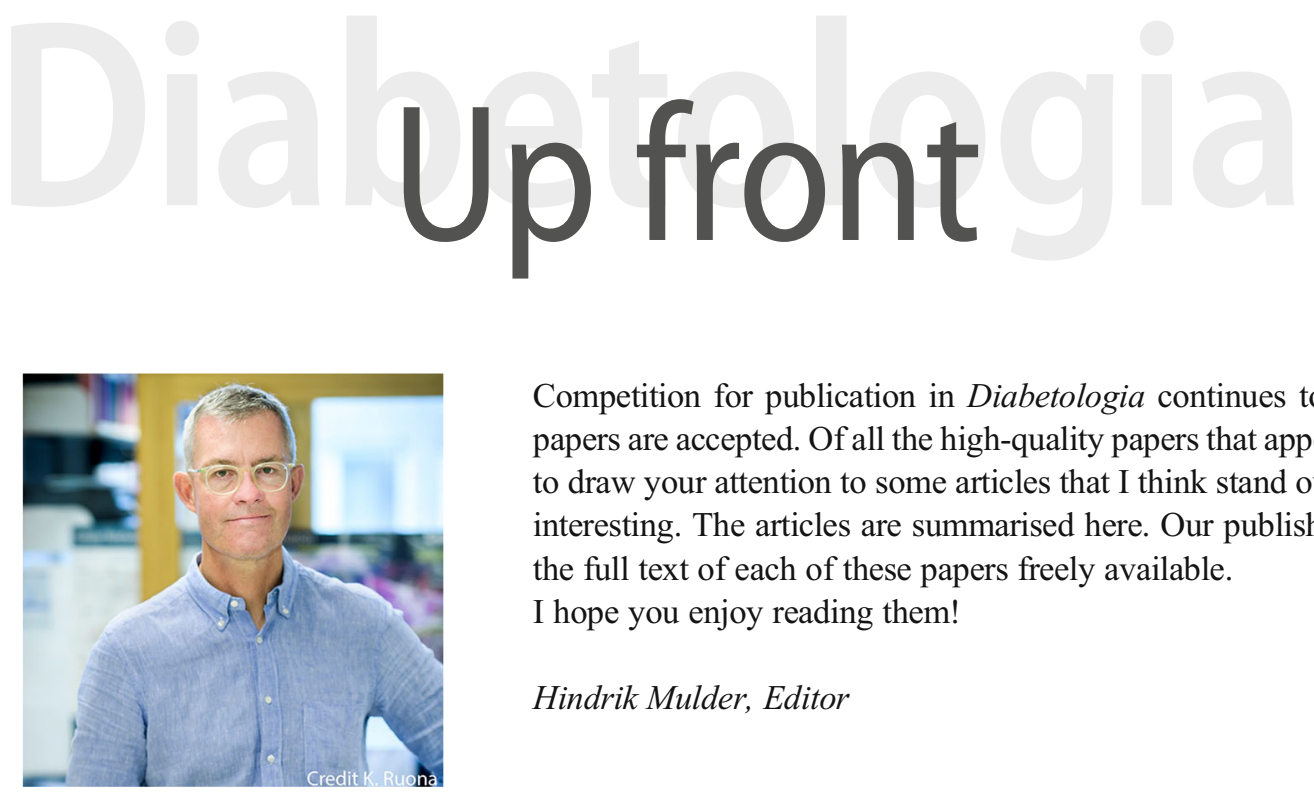

Competition for publication in Diabetologia continues to grow, and less than $20 \%$ of papers are accepted. Of all the high-quality papers that appear in this month's issue I want to draw your attention to some articles that I think stand out in some regard and are very interesting. The articles are summarised here. Our publisher, Springer, has kindly made the full text of each of these papers freely available.

I hope you enjoy reading them!

Hindrik Mulder, Editor

\section{Celebrating 100 years of insulin}

As summarised in an Editorial by our Guest Editor and previous Editor-in-Chief, Sally Marshall (https://doi.org/10.1007/ s00125-021-05417-3), our 2021 special issue (https:// diabetologia-journal.org/collections/insulin-100/) celebrates 100 years since the discovery of insulin. Fralick and Zinman (https://doi.org/10.1007/s00125-020-05371-6) begin by recounting the story of insulin's discovery in Toronto, Canada, in 1921/1922. This discovery meant that type 1 diabetes went from being a death sentence to a chronic condition. Despite this, globally, only one in two people currently have access to the insulin they require; Mbanya and colleagues (https://doi.org/10.1007/s00125-020-05375-2) outline the barriers to insulin access and suggest ways to overcome these. For those fortunate enough to have insulin access, hypoglycaemia remains a frequent complication of insulin therapy. Stephanie Amiel (https://doi.org/10.1007/ s00125-020-05366-3) describes the immediate and cumulative consequences of hypoglycaemia, whilst Rory McCrimmon (https://doi.org/10.1007/s00125-020-05369-0) summarises the impact of recurrent hypoglycaemia on brain function. Chantal Mathieu (https://doi.org/10.1007/s00125020-05354-7) suggests options for minimising hypoglycaemia risk, including patient education, insulin analogues, novel technologies and adjunct therapies. To help us understand how insulin therapies work, Yoon and Diano (https://doi.org/ 10.1007/s00125-021-05395-6) describe the central glucosesensing mechanisms involved in the regulation of glucose metabolism, whilst Kahn et al (https://doi.org/10.1007/ s00125-021-05415-5) outline current and evolving concepts of insulin action/resistance in type 2 diabetes. The authors state that understanding the primary source of metabolic disturbances and drivers of disease will offer important new avenues for the development of novel therapies. Boughton and Hovorka (https://doi.org/10.1007/s00125-021-05391-w) outline an excellent example of a novel therapeutic approach for diabetes, the closed-loop insulin-delivery system, summarising the supporting evidence and limitations of these devices. On a similar note, Weiss and colleagues (https://doi.org/10.1007/ s00125-021-05422-6) introduce us to 'smart' insulin-delivery devices and molecular technologies designed to exploit feedback regulation. As an alternative approach, Douglas Melton (https://doi.org/10.1007/s00125-020-05367-2) explores the promise of stem cell-derived islet replacement therapy, whilst von Herrath and colleagues (https://doi.org/10.1007/s00125021-05398-3) give an overview of therapeutics that may help to prevent the destruction of beta cells in the first place. Turning to diabetes complications, David Nathan (https://doi.org/10. 1007/s00125-021-05397-4) demonstrates that insulin therapy ameliorates the long-term complications of type 1 diabetes, using findings from the DCCT and Epidemiology of Diabetes Interventions and Complications (EDIC) study. To complete this special series, Daniel Drucker (https://doi.org/10.1007/ s00125-021-05396-5) looks to the future, highlighting the potential of smart insulins, fully automated insulin delivery, reprogramming of the immune system and stem cell-derived islets to alter the natural history and treatment of type 1 diabetes. 
This special series is in collaboration with the University of Toronto's Insulin 100 Scientific Symposium (https:// insulin100.com/).

Retinal venular tortuosity and fractal dimension predict incident retinopathy in adults with type 2 diabetes: the Edinburgh Type 2 Diabetes Study

Rachel B. Forster, Emmanuel Sandoval Garcia, Anniek J. Sluiman, Sheila M. Grecian, Stela McLachlan, Tom J. MacGillivray, Mark W. J. Strachan, Jackie F. Price, on behalf of the Edinburgh Type 2 Diabetes Study (ET2DS) investigators

Diabetic retinopathy is a common complication of diabetes and can have a major impact on quality of life. In this issue, Forster et al (https://doi.org/10.1007/s00125-021-05388-5) report that recent developments have allowed for detailed analysis of the retinal vessels in the back of the eye. This gives hope of identifying early changes that can predetermine local vascular disease. Through follow-up of the Edinburgh Type 2 Diabetes Study, the authors aimed to identify associations between several retinal vessel measurements and the development of diabetic retinopathy. They identified that increased venular tortuosity (curvature of the small veins and how much the vessels twist) and decreased fractal dimension (complexity of the vessel system) were both associated with later development of diabetic retinopathy. Further analysis found venular tortuosity to be a possible biomarker candidate for future risk prediction models, which, according to the authors, could be used to help identify risk of diabetic retinopathy prior to disease onset.

\section{Saturated fatty acids entrap PDX1 in stress granules and impede islet beta cell function}

Mu Zhang, Chunjie Yang, Meng Zhu, Li Qian, Yan Luo, Huimin Cheng, Rong Geng, Xiaojun Xu, Cheng Qian, Yu Liu

Failure of pancreatic and duodenal homeobox factor 1 (PDX1) to localise in the nucleus of islet beta cells under high-fat-diet conditions may contribute to beta cell failure in type 2 diabetes; however, the mechanism of PDX1 intracellular mislocalisation is unclear. Saturated fatty acids (SFAs) are known to be adverse factors in blood glucose regulation, but the underlying mechanisms for this are unknown. Owing to their biochemical features, SFAs are involved in liquid-liquid phase separation, whilst stress granules (SGs) are cytoplasmic structures that form by phase separation and impair nucleocytoplasmic transport. In this issue, Zhang et al (https://doi.org/10.1007/s00125-021-05389-4) investigated whether SG formation contributes to PDX1 mislocalisation and beta cell dysfunction under SFA-induced stress conditions. They found that SGs assembled in cells after they were exposed to SFAs, and that this was dependent on the phosphoinositide 3-kinase (PI3K)/eukaryotic translation initiation factor $2 \alpha$ (eIF2 $\alpha$ ) axis. They also demonstrated that PDX1 was entrapped in SGs after SFA treatment. The authors suggest that modulation of SG formation may ameliorate the impairment of pancreatic beta cell function and may be a potential therapeutic strategy for obesity and type 2 diabetes.

Genetic ablation of C-reactive protein gene confers resistance to obesity and insulin resistance in rats

Mengliu Yang, Sheng Qiu, Yirui He, Ling Li, Tong Wu, Ning Ding, Fanghong Li, Allan Z. Zhao, Gangyi Yang

Besides serving as a traditional inflammatory marker in the clinic, C-reactive protein (CRP) is positively associated with the development of obesity, diabetes and cardiovascular diseases. However, genetic evidence is needed to definitively conclude whether CRP directly participates in the regulation of energy and glucose metabolism rather than just being a surrogate marker for these. In this issue, Yang, Qiu et al (https://doi.org/10.1007/s00125-021-05384-9) use a Crp knockout rat model to investigate this question. They found that CRP-deficiency led to a significant reduction in weight gain and food intake, elevated energy expenditure and improved insulin sensitivity after exposure to a high-fat diet. Importantly, it also significantly enhanced the weightreducing effect of leptin. The authors state that this study represents the first line of genetic evidence that CRP is not merely a surrogate blood marker for inflammation and metabolic syndromes, but that it also directly regulates adiposity and insulin sensitivity. They conclude that, clinically, elevated blood CRP levels will negate leptin's actions. Hence, reduction of CRP-induced leptin resistance may be a promising therapeutic strategy for the treatment of obesity and type 2 diabetes.

The disproportionate excess mortality risk of COVID-19 in younger people with diabetes warrants vaccination prioritisation

Andrew P. McGovern, Nick J. Thomas, Sebastian J. Vollmer, Andrew T. Hattersley, Bilal A. Mateen, John M. Dennis

In this issue, McGovern et al (https://doi.org/10.1007/s00125021-05404-8) investigate the combined effects of age and diabetes on Coronavirus disease-2019 (COVID-19) mortality. Triangulating published data from large UK population-based (OpenSAFELY $[n=17,278,392]$ and QCOVID $[n=$ 6,083,102]) and critical care-based (COVID-19 Hospitalisation in England Surveillance System [CHESS] cohort $[n=19,256])$ studies, the authors describe a 
disproportionate excess relative mortality risk in younger people with diabetes compared with older people with diabetes. For example, a person aged 40 years with diabetes has the equivalent COVID-19 mortality risk of a 60-year-old person without diabetes: an additional risk of 20 years. This difference reduces significantly with increasing age, so that a person

All text supplied by the authors. aged 70 with diabetes has the equivalent COVID-19 mortality risk of a 78-year-old person without diabetes: an additional risk of just 8 years. The authors suggest that country-level strategies to define priority groups for vaccination should consider the disproportionate relative risk of COVID-19 mortality in middle-aged people with diabetes.

Publisher's note Springer Nature remains neutral with regard to jurisdictional claims in published maps and institutional affiliations. 